\title{
Avaliação de Herbicidas para o Controle de Egéria em Laboratório, Caixa D'Água e Represa sem FluXo de ÁGUA ${ }^{1}$
}

\author{
Efficacy of Fluridone in Controlling Egeria spp. in Tanks and in a Small Pond Without Water Flow
}

\author{
TANAKA, R.H. ${ }^{2}$, VELINI, E.D. ${ }^{3}$, MARTINS, D. ${ }^{3}$, BRONHARA, A.A. ${ }^{4}$, SILVA, M.A.S. ${ }^{5}$, \\ CAVENAGHI, A.L. ${ }^{5}$ e TOMAZELA, M.S. ${ }^{6}$
}

\begin{abstract}
RESUMO - Este projeto teve por objetivo avaliar a eficácia de herbicidas para controle de plantas aquáticas submersas das espécies Egeria densa e Egeria najas. O estudo foi realizado em três etapas consecutivas: em laboratório, em caixas d'água e numa represa de pequeno porte, sem fluxo de água. Na primeira etapa, avaliou-se a eficiência de 23 herbicidas utilizados em áreas agrícolas e disponíveis no mercado brasileiro. As elevadas concentrações em que os herbicidas foram efetivos inviabilizam programas de controle fundamentados em uma única aplicação. No experimento em caixas d'água, foram avaliados os efeitos de doses crescentes de fluridone, nas formulações líquida e granulada, no controle de Egeria densa e Egeria najas. Foram testadas as concentrações de 10,20,40, 80 e 150 ppb de fluridone na formulação líquida e 20, 40, 80 e 150 ppb na granulada. Após uma única aplicação, os resultados indicaram que o fluridone, nas concentrações de 80 e 150 ppb, em ambas as formulações, foi eficaz no controle dessas duas espécies. No experimento em represa de pequeno porte sem fluxo de água, avaliou-se o efeito da manutenção das concentrações de fluridone no controle de Egeria najas. Uma represa de $1.980 \mathrm{~m}^{2}$ foi tratada com sete aplicações de fluridone, na formulação líquida, visando a manutenção das concentrações do ingrediente ativo na água entre 10 e 20 ppb. Os resultados indicaram que o controle proporcionado pelo herbicida foi superior a $99 \%$. Não houve modificação significativa nas características relacionadas à qualidade da água.
\end{abstract}

Palavras-chave: Egeria densa, Egeria najas, planta aquática, fluridone.

\begin{abstract}
The objective of this research was to evaluate the efficacy of herbicides in controlling the submerged aquatic weeds Egeria densa and Egeria najas. The study was carried out in three consecutive stages: in the laboratory, in tanks and in a small pond without water flow. In the first stage, the efficacy of 23 herbicides used in agriculture and available in Brazil was evaluated. Results showed that none of the herbicides could be used to control these species, if applied just once. In the tank experiment the effect of increasing rates of fluridone (in two formulations: liquid and pellet), on the control of the species Egeria densa and Egeria najas was evaluated. Concentrations of 10,20,40,80 and $150 \mathrm{ppb}$ of the liquid formulation and 20, 40, 80 and $150 \mathrm{ppb}$ of pellet formulations were used. After a single application, results showed that fluridone, at 80 and $150 \mathrm{ppb}$ of both liquid and pellet formulations, was efficient in controlling both species. In the small pond experiment, the effect of keeping fluridone concentrations for an extended period of time on the control of Egeria najas was evaluated. A 1,980 $\mathrm{m}^{2}$ pond was treated seven times to keep herbicide concentrations in water between 10 and $20 \mathrm{ppb}$. Results showed that control was higher than 99\%. No meaningful change in water quality was found during the experiment.
\end{abstract}

Key words: Egeria densa, Egeria najas, fluridone, aquatic plant.

1 Recebido para publicação em 14/11/2001 e na forma revisada em 22/12/2001.

Parte da dissertação do primeiro autor apresentada para obtenção do título de Mestre em Agronomia.

2 Eng.-Agr., M.S., Companhia Energética de São Paulo. Rua da Consolação, 1875, São Paulo/SP, 01301-100. ${ }^{3}$ Prof. Assistente Doutor, Dep. de Produção Vegetal, FCA-UNESP, Caixa Postal 237, Botucatu-SP, 18603-970. ${ }^{4}$ Eng.-Agr., Rua Álvaro Alvares Abreu Silva Filho, 45, 13066-110 Campinas-SP. ${ }^{5}$ Eng.-Agr., Dr., Dep. de Produção Vegetal, FCA-UNESP. ${ }^{6}$ Eng.-Agr., Dr., Comitê de Defesa Sanitária Vegetal, SAA, Av. Brasil, 2340, 13073-001 Campinas-SP. 


\section{INTRODUÇÃO}

O desenvolvimento excessivo de plantas aquáticas tem prejudicado a utilização da água para a navegação, a geração de energia em usinas hidrelétricas e a captação de água para irrigação e consumos humano e animal em diversos países (Hughes, 1976; Burkhalter, 1975; Pitelli, 1998). Para Patton \& Starnes (1970), o fato de ambientes hídricos tornaremse infestados por plantas aquáticas é uma indicação de que a água apresenta um problema de difícil solução, a eutrofização.

Das espécies submersas, as pertencentes à família Hydrocharitaceae estão entre as que provocam os maiores problemas (Fox et al., 1994; Howard-Williams et al., 1996). O gênero Egeria é composto por duas espécies perenes, disseminadas principalmente por reprodução vegetativa: Egeria densa Planch. e Egeria najas Planch. (Cook \& Urmi-König, 1984). O desenvolvimento excessivo destas espécies, juntamente com o Ceratophyllum demersum, tem prejudicado a geração de energia na Usina Hidrelétrica Eng. Souza Dias (Jupiá), no Estado de São Paulo, exigindo a realização de estudos sobre sua biologia e formas de manejo das populações.

Haller (1998), discutindo o controle químico de plantas aquáticas nos Estados Unidos, observou que há apenas seis herbicidas registrados para uso aquático: $2,4-\mathrm{D}$, diquat, cobre, endothall, fluridone e glyphosate. O autor afirmou que, para o controle de E. densa, apenas cobre, diquat e fluridone seriam opções.

No Brasil, o controle de plantas aquáticas submersas tornou-se alvo de estudos intensos nos últimos anos, devido aos prejuízos provocados por esse tipo de vegetação nos reservatórios de usinas hidrelétricas. Outro fato que impulsionou os estudos de manejo de plantas submersas foi o processo de registro do herbicida fluridone no País.

Em diversos países, o fluridone (1-metil3-fenil-5-[3-(trifluorometil)-fenil]-4-(1H)piridinona) é um dos herbicidas mais utilizados no controle de plantas aquáticas, sendo registrado nos EUA para o controle de E. densa e outras espécies submersas.

De acordo com McCowen et al. (1979), o fluridone inibe a biossintese de precursores de carotenóides. Como estes protegem a clorofila da foto-oxidação, a inibição de sua síntese diminui este sistema protetor e a clorofila torna-se suscetível à degradação. O sintoma típico de fluridone em plantas submersas é a clorose das regiões de crescimento, que começa a aparecer entre três e seis semanas após o tratamento.

A aplicação de fluridone para controle de plantas submersas pouco ou nada tem em comum com o controle de plantas terrestres. Para Netherland et al. (1993), a eficácia do fluridone depende do tempo de exposição e da concentração do produto na água.

Fox et al. (1994) concluíram que foi necessária exposição contínua ao fluridone, entre 10 e $15 \mathrm{ppb}$, por um período de 8 a 10 semanas, para se obter controle de $H$. verticillata. A exposição contínua das plantas ao herbicida pode ser atingida com múltiplas aplicações ou com formulações de lenta liberação do ingrediente ativo.

Até o início deste projeto, nenhum trabalho havia sido feito no Brasil visando controle químico dessas espécies. O presente estudo foi desenvolvido entre 1997 e 1999, partindo da avaliação de herbicidas disponíveis no mercado agrícola brasileiro e chegando à avaliação do herbicida fluridone, em subsídio ao seu processo de registro.

\section{MATERIAL E MÉTODOS}

\section{Experimento em laboratório}

Fragmentos apicais de E. densa e E. najas, com cerca de $15 \mathrm{~cm}$ cada, foram coletados no reservatório de Jupiá e mantidos em tubos de ensaio de $250 \mathrm{ml}$ contendo solução de Hoagland a 5\%. Durante os testes, conduzidos em 1997 no laboratório da Faculdade de Ciências Agronômicas da UNESP, em Botucatu, as plantas foram mantidas em condições de laboratório, com a temperatura oscilando entre 22 e $25^{\circ} \mathrm{C}$. A luz ambiente foi suplementada por lâmpadas fluorescentes.

Foram testados os herbicidas: diquat, paraquat, atrazina, diuron, bentazon, propanil, glyphosate (Roundup e Rodeo), sulfosate, glufosinate, clomazone, oxyfluorfen, fomesafen, lactofen, oxadiazon, imazapyr, nicosulfuron, fexonaprop, fluazifop-p-butil, sethoxydim, 
sethoxydim + óleo, alachlor, molinate, 2,4-D, quinclorac e o espalhante adesionante MON 0818. Foram testadas as concentrações de $10.000 ; 1.000 ; 100 ; 10 ; 1 ; 0,1 ;$ e 0,01 ppm dos produtos comerciais.

Foi utilizado o delineamento inteiramente casualizado, com três repetições. As avaliações visuais foram realizadas diariamente até a estabilização dos sintomas de intoxicação das plantas pelos produtos. Adotaram-se os conceitos: injúria severa ou morte da planta; injúria leve ou moderada; sem efeito; e estímulo ao crescimento.

\section{Experimento em caixas d'água}

Este experimento visou avaliar o efeito de concentrações crescentes de fluridone nas formulações líquida e granulada no controle de E. densa e E. najas. Os resultados obtidos subsidiaram a etapa seguinte, indicando a dose e a formulação mais adequadas. O experimento foi instalado em abril de 1998 na Estação de Hidrobiologia e Aqüicultura de Barra Bonita, da Companhia Energética de São Paulo.

Foram utilizadas caixas d'água de fibra de vidro, com 320 litros de volume. Cada caixa recebeu uma camada de 0,2 $\mathrm{m}$ de terra, cujas características físico-químicas são apresentadas na Tabela 1, e uma adubação com 80 gramas da fórmula 4-14-8, antes do plantio. Em cada caixa foram plantadas 20 plantas de cada espécie (coletadas no reservatório de Jupiá), utilizando-se fragmentos apicais de $0,2 \mathrm{~m}$, enterradas a $4 \mathrm{~cm}$ no solo. As plantas foram mantidas por dois meses nos tanques antes da aplicação do herbicida. No momento em que o herbicida foi aplicado, as plantas haviam atingido a superfície da água e já se encontravam estabelecidas.

Cada caixa foi definida como uma parcela experimental. O delineamento experimental utilizado foi o de blocos ao acaso, com quatro repetições. Os tratamentos testados são apresentados na Tabela 2 .

Os herbicidas foram aplicados em 9/6/ 1998, em dose única. Preparou-se uma solução estoque de 1,0 g i.a. $\mathrm{L}^{-1}$ em balão volumétrico, a partir da formulação comercial líquida (Sonar AS, 479 g i.a. $\mathrm{L}^{-1}$ ). Nos tratamentos com a formulação granulada de fluridone (Sonar SRP, $50 \mathrm{~g}$ i.a. $\mathrm{kg}^{-1}$ ), o herbicida foi distribuído diretamente nas caixas.

Tabela 2 - Tratamentos testados no experimento de controle de Egeria spp. em caixas d'água. Barra Bonita-SP, 1998

\begin{tabular}{|c|c|c|}
\hline Tratamento & Formulação & $\begin{array}{c}\text { Concentração } \\
(\mathrm{ppb})\end{array}$ \\
\hline 1. fluridone & suspensão aquosa $^{1 /}$ & 10 \\
\hline 2. fluridone & suspensão aquosa & 20 \\
\hline 3. fluridone & granulada $^{2 /}$ & 20 \\
\hline 4. fluridone & suspensão aquosa & 40 \\
\hline 5. fluridone & granulada & 40 \\
\hline 6. fluridone & suspensão aquosa & 80 \\
\hline 7. fluridone & granulada & 80 \\
\hline 8. fluridone & suspensão aquosa & 150 \\
\hline 9. fluridone & granulada & 150 \\
\hline $\begin{array}{l}\text { 10. testemunha sem } \\
\text { herbicida }\end{array}$ & - & 0 \\
\hline
\end{tabular}

Tabela 1 - Características físico-químicas do solo utilizado no experimento em caixas d'água. Barra Bonita-SP, 1998

\begin{tabular}{|c|c|c|c|c|c|c|c|c|c|}
\hline \multicolumn{10}{|c|}{ Característica química } \\
\hline \multirow{2}{*}{$\begin{array}{l}\mathrm{pH}(\mathrm{em} \\
\left.\mathrm{CaCl}_{2}\right)\end{array}$} & $\begin{array}{l}\text { Matéria } \\
\text { orgânica }\end{array}$ & $\mathrm{P}$ resina & $\mathrm{H}+\mathrm{Al}$ & K & $\mathrm{Ca}$ & $\mathrm{Mg}$ & SB & CTC & $\mathrm{V} \%$ \\
\hline & \multicolumn{2}{|c|}{$\left(\mathrm{g} \mathrm{dm}^{-3}\right)$} & \multicolumn{7}{|c|}{$\left(\mathrm{mmol}_{\mathrm{c}} \mathrm{dm}^{-3}\right)$} \\
\hline 5,2 & 30 & 14 & 72 & 2,1 & 8,0 & 2,5 & 12,6 & 85,0 & 16 \\
\hline \multicolumn{10}{|c|}{ Característica física } \\
\hline \multicolumn{2}{|c|}{$\begin{array}{c}\text { Areia total } \\
(\%)\end{array}$} & & \multicolumn{2}{|c|}{$\begin{array}{c}\text { Argila } \\
(\%)\end{array}$} & \multicolumn{2}{|c|}{$\begin{array}{l}\text { Silte } \\
(\%)\end{array}$} & & \multicolumn{2}{|c|}{ Textura do solo } \\
\hline \multicolumn{2}{|r|}{18} & & \multicolumn{2}{|c|}{70} & \multicolumn{2}{|c|}{12} & & \multicolumn{2}{|c|}{ muito argilosa } \\
\hline
\end{tabular}


As avaliações visuais de toxicidade do herbicida às plantas foram realizadas aos 8 , $15,29,35,51$ e 63 dias após a aplicação, utilizando-se uma escala de 0 (sem sintoma) a 100\% (morte da planta). Avaliou-se, por cromatografia líquida de alto desempenho, a concentração de fluridone em cada caixa, 63 dias após sua aplicação.

Os dados de eficácia de controle foram submetidos à análise de variância pelo teste $\mathrm{F}$, e as comparações das médias dos tratamentos foram feitas pelo teste de Tukey a 5\% de significância. Para a realização das análises, os dados foram transformados em arco seno da raiz quadrada de $\mathrm{X} / 100$.

\section{Experimento em represa de pequeno porte, sem fluxo d'água}

Esta etapa foi conduzida em uma represa rural, localizada no município de Ilha SolteiraSP, infestada por E. najas. A represa apresentava área de $1.980 \mathrm{~m}^{2}$, volume inicial de $1.072,8 \mathrm{~m}^{3}$ e profundidade média de $54 \mathrm{~cm}$. Para a condução do experimento, as entradas e saídas de água da represa foram fechadas.

As aplicações de fluridone, na formulação líquida, foram realizadas caminhando-se pela margem da represa. Para aumentar a uniformidade de distribuição do herbicida, realizouse uma diluição prévia do produto comercial em água.

Na primeira aplicação, realizada em 7/11/ 1998, embora o levantamento preliminar indicasse um volume estimado de $1.021,3 \mathrm{~m}^{3}$, o volume total de água foi aferido utilizandose o próprio fluridone. A concentração inicial desejada era de 20 ppb, mas aplicou-se somente a quantidade necessária para alcançar a concentração de $15 \mathrm{ppb}$. Como a concentração alcançada após esta aplicação foi de 14,28 ppb, o volume foi novamente estimado em $1.072,8 \mathrm{~m}^{3}$ e aplicou-se, em 10/11/98, o volume de herbicida necessário para que a concentração de $20 \mathrm{ppb}$ fosse alcançada.

Durante a segunda quinzena de dezembro, em razão das chuvas, o volume da represa aumentou. O procedimento descrito anteriormente foi repetido estimando-se o novo volume em $1.502 \mathrm{~m}^{3}$, com profundidade de $76 \mathrm{~cm}$, mantida até o final do experimento.
Foram realizadas sete aplicações na represa. Para orientar as reaplicações do herbicida, sua concentração em água era determinada antes e um dia após a aplicação do herbicida. A concentração do fluridone era analisada somente um dia após a aplicação, para que houvesse diluição uniforme na represa, minimizando os erros de amostragem. Os volumes do produto comercial utilizados foram: $32 \mathrm{ml}$ em 6/11/1998; $14 \mathrm{ml} \mathrm{em} \mathrm{9/11/1998;} 35 \mathrm{ml}$ em 24/11/1998; $25 \mathrm{ml} \mathrm{em} \mathrm{9/12/1998;} 40 \mathrm{ml}$ em 26/12/1998; $30 \mathrm{ml} \mathrm{em} \mathrm{6/1/1999;} \mathrm{e} 30 \mathrm{ml}$ em 16/1/1999.

Para definição das quantidades de produto a serem reaplicadas, optou-se pela construção de uma curva de concentração de fluridone em função do tempo. Para isso, foram determinadas as concentrações de fluridone imediatamente após a primeira aplicação e também 8 , 10,14 e 15 dias depois. Os resultados possibilitaram estimar a meia-vida de fluridone na represa, o que permitiu inferir a concentração de fluridone no momento da segunda aplicação. Nas aplicações seguintes, novos dados eram agregados às informações anteriores, aumentando a precisão dos resultados. O modelo linear foi o mais adequado para representar as concentrações de fluridone ao longo do tempo.

Amostras de água foram coletadas antes do início das aplicações e aos $20,35,52,63$, 72 e 87 dias após o início das aplicações. Foram avaliados a condutividade elétrica, $\mathrm{pH}$, sólidos totais dissolvidos, teor de oxigênio dissolvido e turbidez em todos esses períodos. O experimento foi encerrado em $1 / 2 / 99$, aos 87 dias após o início da aplicação do herbicida. No dia 18/3/99, 132 dias após a primeira aplicação, a represa foi visitada para avaliação da infestação por E. najas e para coleta de amostras de água para monitoramento da concentração de fluridone.

\section{RESULTADOS E DISCUSSÃO}

\section{Experimento em laboratório}

Os resultados obtidos para $E$. densa e E. najas são apresentados na Tabela 3. Injúrias severas ou morte total das plantas só foram verificadas em concentrações iguais ou superiores a $10 \mathrm{ppm}$. No caso do diquat, foram verificadas injúrias leves em concentrações de 
até $0,1 \mathrm{ppm}$. As elevadas concentrações em que os herbicidas controlaram as duas espécies inviabilizam seus usos com base em uma única aplicação.

Tabela 3 - Efeito de diversos herbicidas no controle de Egeria densa e Egeria najas. Experimento em laboratório, BotucatuSP, 1997

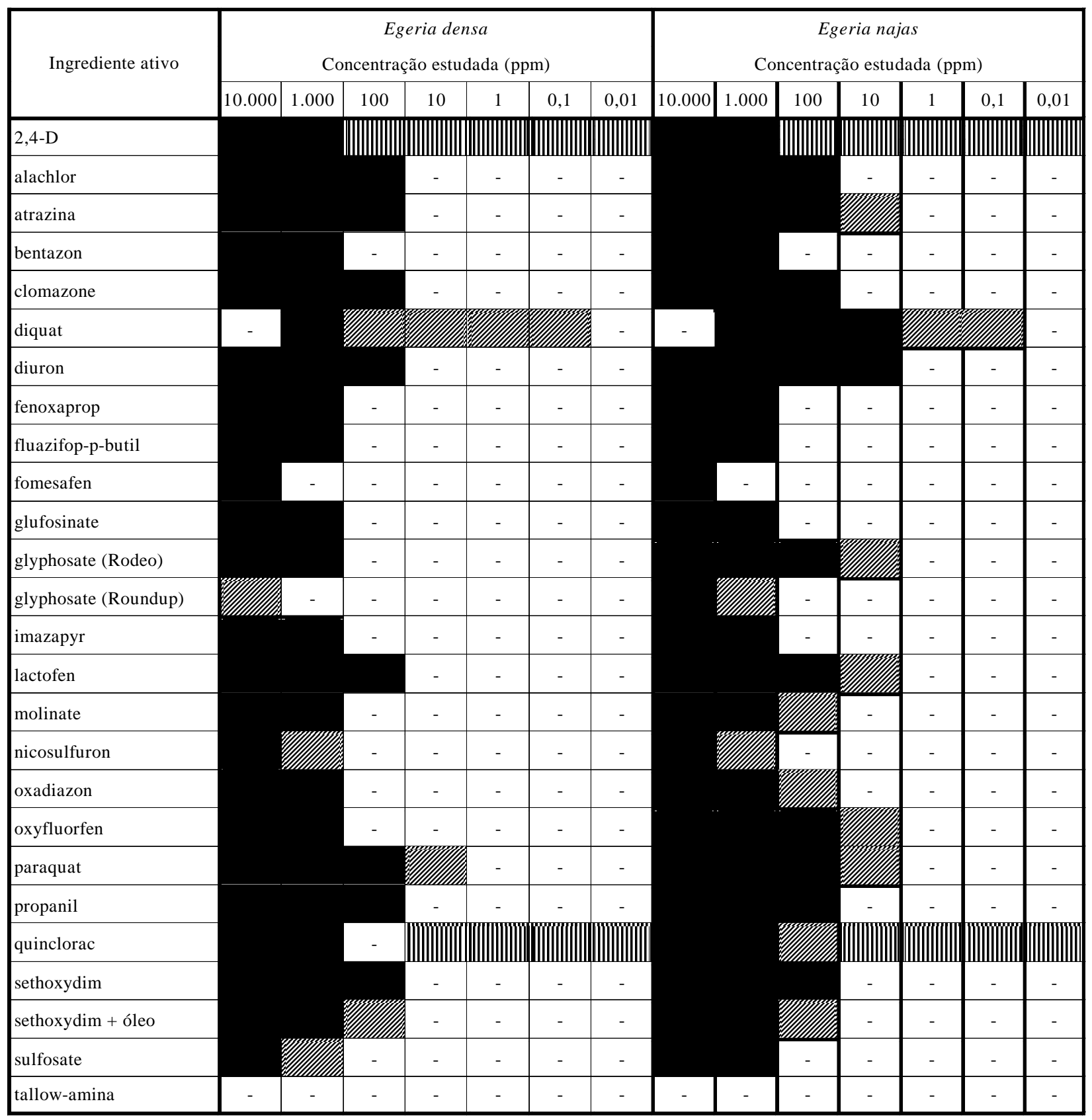

\footnotetext{
Injúria severa ou morte da planta.

Injúria leve ou moderada.

Estímulo ao crescimento.

Sem efeito observável
} 
O 2,4-D provocou morte das plantas nas duas maiores concentrações $(10.000$ e $1.000 \mathrm{ppm}$ ) e estímulo ao crescimento nas demais concentrações. Na Figura 1 é apresentado o crescimento das plantas em função das concentrações deste herbicida. As concentrações de 0,$1 ; 1$; e 10 ppm aumentaram significativamente o comprimento final das plantas de $E$. densa, com máximos efeitos nas concentrações de 0,1 e 1 ppm. Para E. najas, a concentração foi dez vezes maior para que fossem alcançados efeitos similares; o máximo crescimento das plantas ocorreu na concentração de $1 \mathrm{ppm}$.

No caso de E. densa, os estímulos ao crescimento promovidos pelo quinclorac, apresentados na Figura 2, foram superiores aos observados para o 2,4-D, mas ocorreram em concentração dez vezes superior. Para E. najas, os estímulos proporcionados pelo quinclorac foram inferiores aos verificados pelo $2,4-\mathrm{D}$.

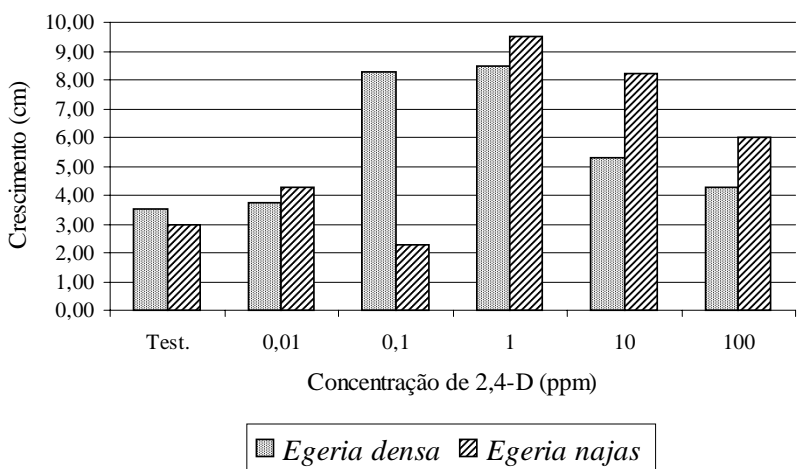

Figura 1 - Efeito da aplicação de 2,4-D sobre o crescimento de E. densa e E. najas. Experimento em laboratório, Botucatu-SP, 1997.

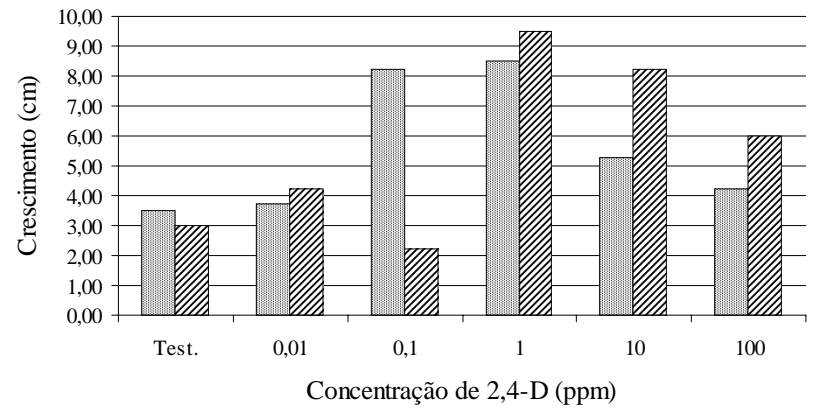

$\square$ Egeria densa Egeria najas

Figura 2 - Efeito da aplicação de quinclorac sobre o crescimento de E. densa e E. najas. Experimento em laboratório, Botucatu-SP, 1997.

\section{Experimento em caixas d'água}

Na Tabela 4 estão apresentados os resultados da avaliação de toxicidade de fluridone às plantas de E. densa. Oito dias após a aplicação, não foram observados sintomas significativos de intoxicação nas plantas. Segundo Arnold (1979), os sintomas iniciais aparecem de três a seis dias após a aplicação, notandose pouco controle até duas a quatro semanas. Aos 15 dias, a formulação granulada mostrouse menos eficaz, como esperado, pois esta foi desenvolvida para lenta liberação.

Aos 63 dias após a aplicação do herbicida, observou-se que os tratamentos com doses de 80 e $150 \mathrm{ppb}$, independentemente da formulação, incrementaram os sintomas de intoxicação às plantas, sendo constatada a morte destas. Nesta última avaliação, nas doses de 20 e $40 \mathrm{ppb}$ da formulação granulada e 10, 20 e $40 \mathrm{ppb}$ da formulação líquida, verificou-se o desenvolvimento normal das plantas.

Observou-se que o controle ou não das plantas dependeu da dose aplicada, não havendo diferença significativa entre os sintomas provocados pelas formulações. A Figura 3 apresenta as concentrações inicial e final de fluridone em cada tratamento. Nota-se que nos tratamentos com concentração inicial de 80 e $150 \mathrm{ppb}$, que provocaram a morte das plantas, a concentração final foi superior a $10 \mathrm{ppb}$.

Na Tabela 5 estão apresentadas as avaliações de toxicidade às plantas de E. najas. Oito dias após a aplicação do herbicida, diferentemente do ocorrido com $E$. densa, já puderam ser observados sintomas de intoxicação por fluridone. Aos 15, 29 e 35 dias, todos os tratamentos provocaram toxicidade às plantas, fato que se repetiu até o final do experimento. Aos 63 dias, observou-se decréscimo no controle nas doses de $20 \mathrm{ppb}$, na formulação granulada, e 10, 20 e 40 ppb, na formulação líquida.

Comparando os efeitos do herbicida nas duas espécies, observou-se que $E$. najas mostrou-se mais sensivel que $E$. densa na primeira avaliação, mas, ao final do experimento, o nível de controle das duas espécies foi semelhante. Como o produto provocou sintomas de intoxicação em doses baixas (10 e 20 ppb), esses resultados foram considerados no experimento em represa sem fluxo d'água, no qual se 
procurou manter uma exposição contínua da planta ao herbicida por um período longo, segundo conceito utilizado por Fox et al. (1994).

\section{Experimento em represa de pequeno porte, sem fluxo de água}

Na Tabela 6 estão apresentados os valores referentes às características da água da represa nas várias avaliações realizadas durante o experimento. Ficou evidente que a decomposição das plantas de $E$. najas não afetou a disponibilidade de oxigênio dissolvido. Os valores de $\mathrm{pH}$, condutividade e sólidos totais dissolvidos mantiveram-se praticamente constantes durante todo o ensaio, não havendo alterações que pudessem ser creditadas ao herbicida. Os valores de turbidez sofreram grandes variações, mas que são relacionadas com a entrada de água proveniente das chuvas.

Tabela 4 - Médias das notas atribuídas nas avaliações visuais de toxicidade de fluridone às plantas de Egeria densa aos 8,15 , 29, 35, 51 e 63 dias após a aplicação. Barra Bonita-SP, 1998

\begin{tabular}{|c|c|c|c|c|c|c|c|}
\hline \multirow{3}{*}{ Tratamento } & \multirow{3}{*}{$\begin{array}{c}\text { Concen- } \\
\text { tração } \\
\text { (ppb) }\end{array}$} & \multicolumn{6}{|c|}{ Nota atribuída ${ }^{3 /}$} \\
\hline & & \multicolumn{6}{|c|}{ Dias após a aplicação } \\
\hline & & 8 & 15 & 29 & 35 & 51 & 63 \\
\hline 1. fluridone, líquido ${ }^{1 /}$ & 10 & $0 \mathrm{a}$ & $20 a b$ & $37 \mathrm{a}$ & $32 \mathrm{a}$ & $47 \mathrm{bc}$ & $27 \mathrm{~cd}$ \\
\hline 2. fluridone, líquido & 20 & $0 \mathrm{a}$ & $30 \mathrm{a}$ & $47 \mathrm{a}$ & $50 \mathrm{a}$ & $55 \mathrm{abc}$ & $47 \mathrm{bc}$ \\
\hline 3. fluridone, granulado ${ }^{2 /}$ & 20 & $0 \mathrm{a}$ & $2 \mathrm{c}$ & $42 \mathrm{a}$ & $37 \mathrm{a}$ & $52 \mathrm{abc}$ & $52 \mathrm{bc}$ \\
\hline 4. fluridone, líquido & 40 & $2 \mathrm{a}$ & $27 \mathrm{a}$ & $37 \mathrm{a}$ & $40 \mathrm{a}$ & $66 \mathrm{ab}$ & $60 \mathrm{bc}$ \\
\hline 5. fluridone, granulado & 40 & $0 \mathrm{a}$ & $2 \mathrm{c}$ & $30 \mathrm{a}$ & $27 \mathrm{a}$ & $57 \mathrm{abc}$ & $85 \mathrm{ab}$ \\
\hline 6. fluridone, líquido & 80 & $2 \mathrm{a}$ & $22 \mathrm{ab}$ & $42 \mathrm{a}$ & $42 \mathrm{a}$ & $62 a b c$ & $100 \mathrm{a}$ \\
\hline 7. fluridone, granulado & 80 & $0 \mathrm{a}$ & $7 \mathrm{bc}$ & $42 \mathrm{a}$ & $45 \mathrm{a}$ & $62 a b c$ & 97 a \\
\hline 8. fluridone, líquido & 150 & $10 \mathrm{a}$ & $27 \mathrm{a}$ & $47 \mathrm{a}$ & $42 \mathrm{a}$ & $72 \mathrm{a}$ & $100 \mathrm{a}$ \\
\hline 9. fluridone, granulado & 150 & $0 \mathrm{a}$ & $25 \mathrm{a}$ & $40 \mathrm{a}$ & $37 \mathrm{a}$ & $62 a b c$ & $100 \mathrm{a}$ \\
\hline 10. testemunha & 0 & $0 \mathrm{a}$ & $0 \mathrm{c}$ & $0 \mathrm{~b}$ & $0 \mathrm{~b}$ & $0 \mathrm{~d}$ & $0 \mathrm{~d}$ \\
\hline F tratamentos & & $1,68 \mathrm{~ns}$ & $19,46 * *$ & $7,70 * *$ & $7,05 * *$ & $45,78 * *$ & $20,32 * *$ \\
\hline F blocos & & $1,09 \mathrm{~ns}$ & $1,31 \mathrm{~ns}$ & $0,20 \mathrm{~ns}$ & $0,26 \mathrm{~ns}$ & $3,57 *$ & $3,41 *$ \\
\hline d.m.s. $5 \%$ & & 15,9 & 14,5 & 22,6 & 23,5 & 12,0 & 32,9 \\
\hline $\mathrm{CV}(\%)$ & & 294,5 & 29,3 & 26,2 & 27,9 & 10,9 & 22,2 \\
\hline
\end{tabular}

${ }^{1 /}\left(41,7 \%\right.$ i.a. em peso; 479 g i.a. $\left.\mathrm{L}^{-1}\right) ;{ }^{2 /}\left(5 \%\right.$ i.a. em peso; $50 \mathrm{~g}$ i.a. $\left.\mathrm{kg}^{-1}\right)$; e ${ }^{3 /}$ Conforme escala: $0 \%$ (planta sem sintomas) a $100 \%$ (planta morta).

ns não-significativo; * significativo a $5 \%$ de probabilidade; e $* *$ significativo a $1 \%$ de probabilidade.

Médias seguidas da mesma letra, na coluna, não diferem estatisticamente entre si pelo teste de Tukey a 5\% de probabilidade.

Para realização da análise estatística, os dados foram transformados em arco seno da raiz quadrada de X/100.

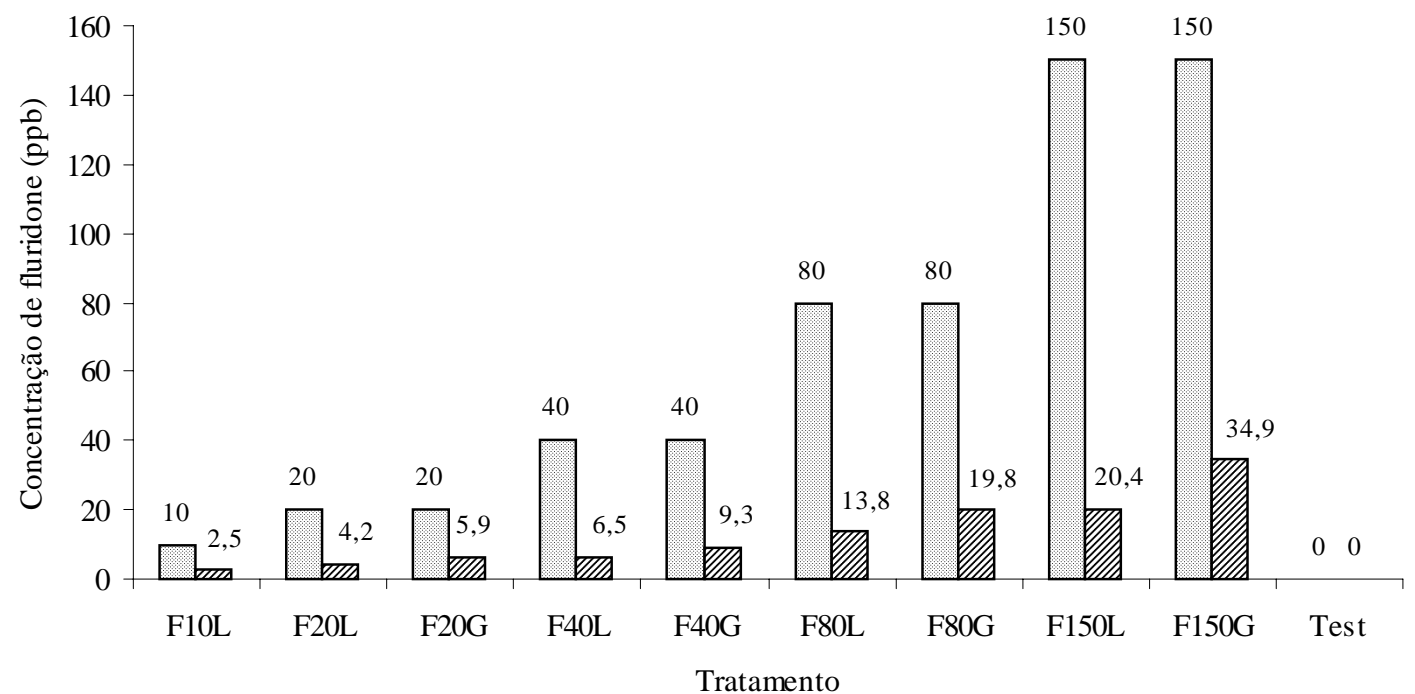

Figura 3 - Concentrações inicial e final de fluridone no experimento em caixas d'água. Barra Bonita-SP, 1998. 
Tabela 5 - Médias das notas atribuídas nas avaliações visuais de toxicidade de fluridone às plantas de Egeria najas aos 8, 15, 29, 35, 51 e 63 dias após a sua aplicação. Barra Bonita-SP, 1998

\begin{tabular}{|c|c|c|c|c|c|c|c|}
\hline \multirow{3}{*}{ Tratamento } & \multirow{3}{*}{$\begin{array}{c}\text { Concen- } \\
\text { tração } \\
(\mathrm{ppb})\end{array}$} & \multicolumn{6}{|c|}{ Nota atribuída $a^{\frac{3}{}}$} \\
\hline & & \multicolumn{6}{|c|}{ Dias após a aplicação } \\
\hline & & 8 & 15 & 29 & 35 & 51 & 63 \\
\hline 1. fluridone, líquido ${ }^{\underline{1}}$ & 10 & $2 \mathrm{bc}$ & 32 bcd & $45 \mathrm{a}$ & $40 \mathrm{a}$ & $57 \mathrm{bc}$ & $40 \mathrm{bc}$ \\
\hline 2. fluridone, líquido & 20 & $22 a b$ & $50 \mathrm{ab}$ & $62 \mathrm{a}$ & $67 \mathrm{a}$ & $65 a b c$ & $47 \mathrm{~b}$ \\
\hline 3. fluridone, granulado ${ }^{2 /}$ & 20 & $5 a b c$ & $20 \mathrm{~d}$ & $42 \mathrm{a}$ & $52 \mathrm{a}$ & $52 \mathrm{c}$ & $50 \mathrm{~b}$ \\
\hline 4. fluridone, líquido & 40 & $22 \mathrm{ab}$ & $55 \mathrm{a}$ & $42 \mathrm{a}$ & $47 \mathrm{a}$ & $73 \mathrm{ab}$ & $45 \mathrm{~b}$ \\
\hline 5. fluridone, granulado & 40 & 7 abc & $30 \mathrm{~cd}$ & $40 \mathrm{~b}$ & $42 \mathrm{a}$ & $67 \mathrm{abc}$ & $85 \mathrm{ab}$ \\
\hline 6. fluridone, líquido & 80 & $17 \mathrm{ab}$ & $43 \mathrm{abc}$ & $45 \mathrm{a}$ & $52 \mathrm{a}$ & $72 \mathrm{abc}$ & $100 \mathrm{a}$ \\
\hline 7. fluridone, granulado & 80 & $15 \mathrm{abc}$ & $27 \mathrm{~cd}$ & $52 \mathrm{a}$ & $57 \mathrm{a}$ & $72 a b c$ & 97 a \\
\hline 8. fluridone, líquido & 150 & $27 \mathrm{a}$ & $50 \mathrm{ab}$ & $52 \mathrm{a}$ & $52 \mathrm{a}$ & $82 \mathrm{a}$ & $100 \mathrm{a}$ \\
\hline 9. fluridone, granulado & 150 & $17 \mathrm{ab}$ & 35 bcd & $45 \mathrm{a}$ & $45 \mathrm{a}$ & $72 a b c$ & $100 \mathrm{a}$ \\
\hline 10. testemunha & 0 & $0 \mathrm{c}$ & $0 \mathrm{e}$ & $0 \mathrm{~b}$ & $0 \mathrm{~b}$ & $0 \mathrm{~d}$ & $0 \mathrm{c}$ \\
\hline $\mathrm{F}$ tratamentos & & $5,02 * *$ & $33,65 * *$ & $8,09 * *$ & $7,93 * *$ & $49,86 * *$ & $13,79 * *$ \\
\hline F blocos & & $0,96 \mathrm{~ns}$ & $0,56 \mathrm{~ns}$ & $0,11 \mathrm{~ns}$ & $0,34 \mathrm{~ns}$ & $1,48 \mathrm{~ns}$ & $3,45 *$ \\
\hline d.m.s. $5 \%$ & & 23,8 & 11,6 & 24,5 & 26,2 & 12,8 & 39,9 \\
\hline C.V. (\%) & & 55,1 & 14,0 & 25,8 & 26,4 & 10,4 & 27,4 \\
\hline
\end{tabular}

${ }^{1 /}\left(41,7 \%\right.$ i.a. em peso; 479 g i.a. $\left.\mathrm{L}^{-1}\right) \stackrel{2}{2} /^{2}\left(5 \%\right.$ i.a. em peso; 50 g i.a. $\left.\mathrm{kg}^{-1}\right)$; e ${ }^{\underline{3} /}$ Conforme escala: $0 \%$ (planta sem sintomas) a $100 \%$ (planta morta).

ns não-significativo; * significativo a $5 \%$ de probabilidade; e $* *$ significativo a $1 \%$ de probabilidade.

Médias seguidas da mesma letra, na coluna, não diferem estatisticamente entre si pelo teste de Tukey a 5\% de probabilidade.

Para realização da análise estatística, os dados foram transformados em arco seno da raiz quadrada de X/100.

Tabela 6 - Teores de oxigênio, $\mathrm{pH}$, condutividade elétrica, sólidos totais dissolvidos e turbidez da água da represa de pequeno porte, durante o período do experimento. Ilha Solteira-SP, 1998/99

\begin{tabular}{|c|c|c|c|c|c|}
\hline Dias & $\begin{array}{c}\mathrm{O}_{2} \\
(\mathrm{ppm})\end{array}$ & $\mathrm{pH}$ & $\begin{array}{c}\text { Condutividade } \\
\left(\mu \mathrm{S} \mathrm{cm}^{-1}\right)\end{array}$ & $\begin{array}{c}\text { Sólidos Totais } \\
\text { Dissolvidos } \\
(\mathrm{ppm})\end{array}$ & $\begin{array}{c}\text { Turbidez } \\
(\mathrm{FTU})\end{array}$ \\
\hline 0 & 3,43 & 7,11 & 196 & 95 & 4,20 \\
20 & 3,22 & 7,01 & 199 & 101 & 3,85 \\
35 & 3,35 & 7,02 & 185 & 94 & 3,47 \\
52 & 3,58 & 6,95 & 204 & 102 & 6,28 \\
63 & 3,85 & 7,05 & 207 & 104 & 5,85 \\
72 & 4,15 & 7,08 & 205 & 97 & 4,67 \\
87 & 4,35 & 7,04 & 198 & 98 & 3,15 \\
\hline
\end{tabular}

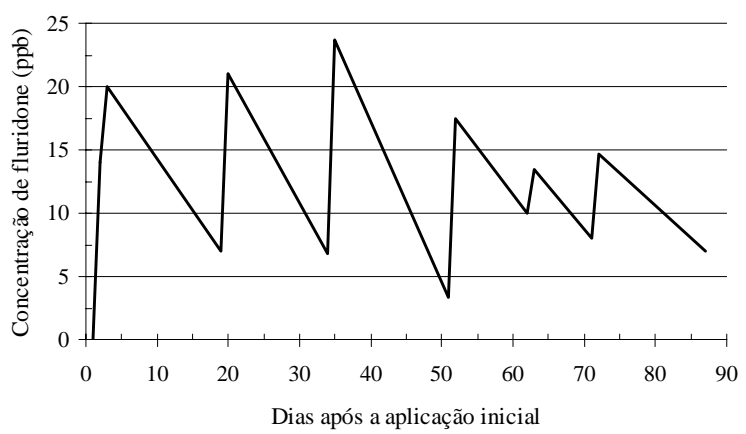

Figura 4 - Concentrações de fluridone na represa de pequeno porte durante o período experimental. Ilha Solteira-SP, 1998/99.
A degradação do fluridone foi da ordem de $4 \%$ a cada dia e meia-vida de 11,8 dias, valor baixo comparado aos obtidos por Netherland et al. (1993). Esse fato pode estar relacionado com a pequena profundidade da represa, o que facilitaria a fotodecomposição do composto. Na Figura 4 são representadas as concentrações de fluridone na água no período de condução do experimento.

Verificou-se que o controle da planta foi completo, não tendo sido coletadas plantas nas amostragens realizadas aos 87 dias após o início do experimento. Avaliações realizadas aos 132 dias, percorrendo-se a represa, indicaram o controle da espécie como sendo superior a 99\%, sendo detectadas apenas duas plantas vivas.

Os resultados obtidos neste experimento permitiram inferir que a manutenção da concentração de fluridone na água entre 3,4 e 23,6 ppb, por um longo período, proporcionou controle total de Egeria najas. Ressalta-se que grande parte dos estudos de controle é proveniente dos EUA, onde espécies como $E$. densa são exóticas, e os programas de manejo têm por objetivo obter o menor nível de infestação de forma economicamente viável. Assim, as recomendações são definidas visando reduzir 
drasticamente as infestações, erradicando-as quando possivel. No Brasil, E. densa e E. najas são espécies nativas. Nestes casos, deve-se considerar que inúmeras espécies de peixes, insetos, aves e outros organismos dependem destas plantas para sua sobrevivência, sendo indesejável a eliminação completa da vegetação. O manejo de plantas aquáticas deve, então, ser planejado visando minimizar os impactos provocados pelas plantas, mantendo-as em áreas onde seus aspectos benéficos suplantem seus impactos negativos.

\section{LITERATURA CITADA}

ARNOLD, W. R. Fluridone - a new aquatic herbicide. J. Aquatic. Plant Manag., v. 17, p. 30-3, 1979.

BURKHALTER, A. P. The State of Florida aquatic weed control program. In: SYMPOSIUM ON WATER QUALITY AND MANAGEMENT THROUGH BIOLOGICAL CONTROL, 1975, Gainesville. Proceedings... Gainesville: 1975. p. $15-19$.

COOK, C. D. K.; URMI-KÖNIG, K. A revision of the genus Egeria (Hydrocharitaceae). i., v. 19, p. 73-96, 1984.

FOX, A.M.; HALLER, W.T.; SHILLING, D. G. Use of fluridone for hydrilla management in the Withlacoochee River, Florida. J. Aquatic. Plant. Manag., v. 32, p. 47-55, 1994.
HALLER, W. T. Options for mechanical and chemical aquatic weed control. In: WORKSHOP CONTROLE DE PLANTAS AQUÁTICAS, 1998, Brasília. Resumos... Brasília: Instituto Brasileiro de Meio Ambiente e dos Recursos Naturais Renováveis, 1998. p. 46-53.

HOWARD-WILLIAMS, C. et al. Patterns of aquatic weed regrowth following mechanical harvesting in New Zealand hydro-lakes. Hydrobiologia, v. 340, n. 1-3, p. 229-34, 1996.

HUGHES, R. W. Research into aquatic weeds in New Zealand waterways: a review. Inf. Series, n. 116, 34 p., 1976.

McCOWEN, M. et al. Fluridone, a new herbicide for aquatic plant management. J. Aquatic. Plant Manag., v. 17 , p. $27-30,1979$.

NETHERLAND, M. D.; GETSINGER, K. D.; TURNER, E. G. Fluridone concentration and exposure time requirements for control of Eurasian watermilfoil and hydrilla. J. Aquatic. Plant Manag., v. 31, p. 189-194, 1993.

PATTON, V. D.; STARNES, W.E. Aquatic weeds and water pollution. Hyacinth Control J., v. 8, n. 2, p. 48-49, 1970.

PITELLI, R. A. Macrófitas aquáticas no Brasil, na condição de problemáticas. In: WORKSHOP CONTROLE DE PLANTAS AQUÁTICAS, 1998, Brasília. Resumos... Brasília: Instituto Brasileiro de Meio Ambiente e dos Recursos Naturais Renováveis, 1998. p. 12-5. 